Research Article

\title{
Fuzzy Conformable Fractional Differential Equations
}

\author{
Atimad Harir (D), Said Melliani, and Lalla Saadia Chadli \\ Laboratory of Applied Mathematics and Scientific Computing, Sultan Moulay Slimane University, P.O. Box 523, \\ Beni Mellal 23000, Morocco \\ Correspondence should be addressed to Atimad Harir; atimad.harir@gmail.com
}

Received 23 October 2020; Revised 2 January 2021; Accepted 27 January 2021; Published 4 February 2021

Academic Editor: Peiguang Wang

Copyright (C) 2021 Atimad Harir et al. This is an open access article distributed under the Creative Commons Attribution License, which permits unrestricted use, distribution, and reproduction in any medium, provided the original work is properly cited.

In this study, fuzzy conformable fractional differential equations are investigated. We study conformable fractional differentiability, and we define fractional integrability properties of such functions and give an existence and uniqueness theorem for a solution to a fuzzy fractional differential equation by using the concept of conformable differentiability. This concept is based on the enlargement of the class of differentiable fuzzy mappings; for this, we consider the lateral Hukuhara derivatives of order $q \in(0,1]$.

\section{Introduction}

Fractional calculus is generalization of differentiation and integration to an arbitrary order. The derivative for fuzzy-valued mappings was developed by [1] that generalized and extended the concept of Hukuhara differentiability ( $H$-derivative) for setvalued mappings to the class of fuzzy mappings. Subsequently, using the $H$-derivative $[2,3]$ started to develop a theory for FDE. The concept of the fuzzy fractional derivative was introduced by [4] and developed by [5-11], but these researchers tried to put a definition of a fuzzy fractional derivative. Most of them used an integral from the fuzzy fractional derivative, two of which are the most popular ones, Riemann-Liouville definition and Caputo definition [12-14]. All definitions above satisfy the property that the fuzzy fractional derivative is linear. This is the only property inherited from the first fuzzy derivative by all of the definitions. However, the following are some of the setbacks of the other definitions [15]. The fuzzy conformable derivative may facilitate some computations:

(i) It satisfies all concepts and rules of an ordinary derivative such as quotient, product, and chain rules while the other fractional definitions fail to meet these rules

(ii) It can be extended to solve exactly and numerically fractional differential equations and systems easily and efficiently
And it was introduced and developed in [16, 17]. The objective of this study is to present some results for fuzzy conformable differentiability and fuzzy fractional integrability of such functions; we study the fuzzy fractional differential equations (FFDEs) by using this derivative and give an existence and uniqueness theorem for a solution of FFDEs.

\section{Preliminaries}

Let us denote by $\mathbb{R}_{\mathscr{F}}=\{u: \mathbb{R} \longrightarrow[0,1]\}$ the class of fuzzy subsets of the real axis satisfying the following properties:

(i) $u$ is normal, i.e, there exists $x_{0} \in \mathbb{R}$ such that $u\left(x_{0}\right)=1$

(ii) $u$ is fuzzy convex, i.e, for $x, y \in \mathbb{R}$ and $0<\lambda \leq 1$,

$$
u(\lambda x+(1-\lambda) y) \geq \min [u(x), u(y)]
$$

(iii) $u$ is upper semicontinuous

(iv) $[u]^{0}=c l\{x \in \mathbb{R} \mid u(x)>0\}$ is compact

Then, $\mathbb{R}_{\mathscr{F}}$ is called the space of fuzzy numbers. Obviously, $\mathbb{R} \subset \mathbb{R}_{\mathscr{F}}$. For $0<\alpha \leq 1$, denote $[u]^{\alpha}=\{x \in \mathbb{R} \mid$ $u(x) \geq \alpha\}$; then, from (i) to (iv), it follows that the $\alpha$-level set $[u]^{\alpha} \in P_{K}(\mathbb{R})$ for all $0 \leq \alpha \leq 1$ is a closed bounded interval which is denoted by $[u]^{\alpha}=\left[u_{1}^{\alpha}, u_{2}^{\alpha}\right]$. By $P_{K}(\mathbb{R})$, we denote 
the family of all nonempty compact convex subsets of $\mathbb{R}$ and define the addition and scalar multiplication in $P_{K}(\mathbb{R})$ as usual.

Theorem 1 (see [7]). If $u \in \mathbb{R}_{\mathscr{F}}$, then

(i) $[u]^{\alpha} \in P_{K}(\mathbb{R})$ for all $0 \leq \alpha \leq 1$

(ii) $[u]^{\alpha_{2}} \subset[u]^{\alpha_{1}}$ for all $0 \leq \alpha_{1} \leq \alpha_{2} \leq 1$

(iii) $\left\{\alpha_{k}\right\} \subset[0,1]$ is a nondecreasing sequence which converges to $\alpha$, and then,

$$
[u]^{\alpha}=\bigcap_{k \geq 1}[u]^{\alpha_{k}} .
$$

Conversely, if $A_{\alpha}=\left\{\left[u_{1}^{\alpha}, u_{2}^{\alpha}\right] ; \alpha \in(0,1]\right\}$ is a family of closed real intervals verifying (i) and (ii), then $\left\{A_{\alpha}\right\}$ defined a fuzzy number $u \in \mathbb{R}_{\mathscr{F}}$ such that $[u]^{\alpha}=A_{\alpha}$ for $0<\alpha \leq 1$ and $[u]^{0}=\overline{\cup_{0<\alpha \leq 1} A_{\alpha}} \subset A_{0}$.

Lemma 1 (see [18]). Let $u, v: \mathbb{R}_{\mathscr{F}} \longrightarrow[0,1]$ be the fuzzy sets. Then, $u=v$ if and only if $[u]^{\alpha}=[v]^{\alpha}$ for all $\alpha \in[0,1]$.

The following arithmetic operations on fuzzy numbers are well known and frequently used below. If $u, v \in \mathbb{R}_{\mathscr{F}}$, then

$$
\begin{aligned}
{[u+v]^{\alpha} } & =\left[u_{1}^{\alpha}+v_{1}^{\alpha}, u_{2}^{\alpha}+v_{2}^{\alpha}\right], \\
& {[\lambda u]^{\alpha}=\lambda[u]^{\alpha}= \begin{cases}{\left[\lambda u_{1}^{\alpha}, \lambda u_{2}^{\alpha}\right],} & \text { if } \lambda \geq 0, \\
{\left[\lambda u_{2}^{\alpha}, \lambda u_{1}^{\alpha}\right],} & \text { if } \lambda<0 .\end{cases} }
\end{aligned}
$$

Definition 1 (see $[19,20]$ ). Let $u, v \in \mathbb{R}_{\mathscr{F}}$. If there exists $w \in \mathbb{R}_{\mathscr{F}}$ such as $u=v+w$, then $w$ is called the $H$-difference of $u, v$, and it is denoted as $u \ominus v$.

Definition 2 (see [21]). Let we denote

$$
\overline{0}= \begin{cases}1, & t=0, \\ 0, & t \neq 0 .\end{cases}
$$

Define $d: \mathbb{R}_{\mathscr{F}} \times \mathbb{R}_{\mathscr{F}} \longrightarrow \mathbb{R}_{+} \cup\{0\}$ by the equation

$$
d(u, v)=\sup _{\alpha \in[0,1]} d_{H}\left([u]^{\alpha},[v]^{\alpha}\right), \quad \text { for all } u, v \in \mathbb{R}_{\mathscr{F}},
$$

where $d_{H}$ is the Hausdorff metric.

$$
d_{H}\left([u]^{\alpha},[v]^{\alpha}\right)=\max \left\{\left|u_{1}^{\alpha}-v_{1}^{\alpha}\right|,\left|u_{2}^{\alpha}-v_{2}^{\alpha}\right|\right\} .
$$

It is well known that $\left(\mathbb{R}_{\mathscr{F}}, d\right)$ is a complete metric space. We list the following properties of $d(u, v)$ :

$$
\begin{aligned}
& d(u+w, v+w)=d(u, v), \\
& d(u, v)=d(v, u), \\
& d(k u, k v)=|k| d(u, v), \\
& d(u, v) \leq d(u, w)+d(w, v),
\end{aligned}
$$

for all $u, v, w \in \mathbb{R}_{\mathscr{F}}$ and $\lambda \in \mathbb{R}$.

Let $\left(A_{k}\right)$ be a sequence in $P_{K}(\mathbb{R})$ converging to $A$. Then, theorem in [2] gives us an expression for the limit.
Theorem 2 (see[2]). If $d\left(A_{k}, A\right) \longrightarrow 0$ as $k \longrightarrow \infty$, then

$$
A=\bigcap_{k \geq 1} \overline{\cup_{m \geq k} A_{m}} \text {. }
$$

\section{Fuzzy Conformable Fractional Differentiability and Fuzzy Fractional Integral}

3.1. Fuzzy Conformable Fractional Differentiability. Now, we present our new definition, which is the simplest and most natural and efficient definition of fractional derivative of order $q \in(0,1]$.

Definition 3 (see[17]). Let $F:(0, a) \longrightarrow \mathbb{R}_{\mathscr{F}}$ be a fuzzy function, and $q^{\text {th }}$ order fuzzy conformable fractional derivative of $F$ is defined by

$$
\begin{aligned}
T_{q}(F)(t) & =\lim _{\varepsilon \longrightarrow 0^{+}} \frac{F\left(t+\varepsilon t^{1-q}\right) \ominus F(t)}{\varepsilon} \\
& =\lim _{\varepsilon \longrightarrow 0^{+}} \frac{F(t) \ominus F\left(t-\varepsilon t^{1-q}\right)}{\varepsilon},
\end{aligned}
$$

for all $t>0, q \in(0,1)$. Let $F^{(q)}(t)$ stands for $T_{q}(F)(t)$. Hence,

$$
\begin{aligned}
F^{(q)}(t) & =\lim _{\varepsilon \longrightarrow 0^{+}} \frac{F\left(t+\varepsilon t^{1-q}\right) \ominus F(t)}{\varepsilon} \\
& =\lim _{\varepsilon \longrightarrow 0^{+}} \frac{F(t) \ominus F\left(t-\varepsilon t^{1-q}\right)}{\varepsilon} .
\end{aligned}
$$

If $F$ is $q$-differentiable in some $(0, a)$ and $\lim _{t \rightarrow 0^{+}} F^{(q)}(t)$ exists, then

$$
F^{(q)}(0)=\lim _{t \longrightarrow 0^{+}} F^{(q)}(t)
$$

and the limits (in the metric $d$ ).

Remark 1. From the definition, it directly follows that if $F$ is $q$-differentiable, then the multivalued mapping $F_{\alpha}$ is $q$-differentiable for all $\alpha \in[0,1]$ and

$$
T_{q} F_{\alpha}=\left[F^{(q)}(t)\right]^{\alpha} \text {. }
$$

where $T_{q} F_{\alpha}$ is denoted from the conformable fractional derivative of $F_{\alpha}$ of order $q$.

Theorem 3 (see[17]). Let $F:(0, a) \longrightarrow \mathbb{R}_{\mathscr{F}}$ be q-differentiable. Denote $F_{\alpha}(t)=\left[f_{1}^{\alpha}(t), f_{2}^{\alpha}(t)\right], \alpha \in[0,1]$. Then, $f_{1}^{\alpha}(t)$ and $f_{2}^{\alpha}(t)$ are $q$-differentiable and

$$
\left[F^{(q)}(t)\right]^{\alpha}=\left[\left(f_{1}^{\alpha}\right)^{(q)}(t),\left(f_{2}^{\alpha}\right)^{(q)}(t)\right]
$$

Theorem 4. Let $F:(0, a) \longrightarrow \mathbb{R}_{\mathscr{F}}$ is q-differentiable on $(0, a)$. If $t_{1}, t_{2} \in(0, a)$ with $t_{1} \leq t_{2}$, then there exists $\lambda \in \mathbb{R}_{\mathscr{F}}$ such that $F\left(t_{2}\right)=F\left(t_{1}\right)+\lambda$. 
Proof. For each $s \in\left[t_{1}, t_{2}\right]$, there exists $\delta(s)>0$ such that the $H$-differences $F\left(s+\varepsilon s^{1-q}\right) \ominus F(s)$ and $F(s) \ominus F\left(s-\varepsilon s^{1-q}\right)$ exist for all $0 \leq \varepsilon<\delta(s)$. Then, we can find a finite sequence $t_{1}=s_{1}<s_{2}<\cdots<s_{n}=t_{2}$ such that the family $\left\{I_{s_{i}}=\left(s_{i}-\delta\left(s_{i}\right), s_{i}+\delta\left(s_{i}\right)\right) \mid i=1,2, \ldots, n\right\}$ covers $\left[t_{1}, t_{2}\right]$ and $I_{s_{i}} \cap I_{s_{i+1}} \neq \varnothing$. Pick $x_{i} \in I_{s_{i}} \cap I_{s_{i+1}}, i=1,2, \ldots, n-1$, such that $s_{i}<x_{i}<s_{i+1}$. Then,

$$
\begin{aligned}
F\left(s_{i+1}\right) & =F\left(x_{i}\right)+A_{1}=F\left(s_{i}\right)+A_{2}+A_{1} \\
& =F\left(s_{i}\right)+\lambda_{i}, \quad i=1,2, \ldots, n-1,
\end{aligned}
$$

for some $A_{1}, A_{2}, \lambda_{i} \in \mathbb{R}_{\mathscr{F}}$. Hence,

$$
F\left(t_{2}\right)=F\left(t_{1}\right)+\sum_{i=1}^{n-1} \lambda_{i}=F\left(t_{1}\right)+\lambda
$$

Theorem 5. If $F:(0, a) \longrightarrow \mathbb{R}_{\mathscr{F}}$ is $q$-differentiable, then it is continuous.

Proof. Let $t, t+t^{1-q} \mathcal{\varepsilon} \in(0, a)$ with $\varepsilon>0$. Then, by properties of equation (7) and the triangle inequality, we have

$$
d\left(F\left(t+t^{1-q} \varepsilon\right), F(t)\right)=d\left(F\left(t+t^{1-q} \varepsilon\right) \ominus F(t), \overline{0}\right) \leq \varepsilon d\left(\frac{\left(F\left(t+t^{1-q} \varepsilon\right) \ominus F(t)\right)}{\varepsilon}, F^{(q)}(t)\right)+\varepsilon d\left(F^{(q)}(t), \overline{0}\right),
$$

where $\varepsilon$ is so small that the $H$-difference $F\left(t+t^{1-q} \mathcal{E}\right) \ominus F(t)$ exists. By the differentiability, the right-hand side goes to zero as $\varepsilon \longrightarrow 0^{+}$, and hence, $F$ is right continuous. The left continuity is proved similarly.

Theorem 6. Let $q \in(0,1]$. If $F$ is differentiable and $F$ is $q$-differentiable, then

$$
T_{q} F(t)=t^{1-q} F^{\prime}(t)
$$

The proof is similar to the proof of Theorem 8 case (i) in [17] and is omitted.

Theorem 7. Let $q \in(0,1]$, and if $F, G:(0, a) \longrightarrow \mathbb{R}_{\mathscr{F}}$ are $q$-differentiable and $\lambda \in \mathbb{R}$, then

$$
\begin{aligned}
& T_{q}(F+G)(t)=T_{q}(F)+T_{q}(G) \text { and } \\
& T_{q}(\lambda F)(t)=\lambda T_{q}(F)(t)
\end{aligned}
$$

Proof. Since $F$ is $q$-differentiable, it follows that $F\left(t+\varepsilon t^{1-q}\right) \ominus F(t)$ exists, i.e., there exists $u_{1}\left(t, \varepsilon t^{1-q}\right)$ such that

$$
F\left(t+\varepsilon t^{1-q}\right)=F(t)+u_{1}\left(t, \varepsilon t^{1-q}\right)
$$

Analogously, since $G$ is $q$-differentiable, there exists $v_{1}\left(t, \varepsilon t^{1-q}\right)$ such that

$$
G\left(t+\varepsilon t^{1-q}\right)=G(t)+v_{1}\left(t, \varepsilon t^{1-q}\right)
$$

and we get

$$
\begin{aligned}
F\left(t+\varepsilon t^{1-q}\right)+G\left(t+\varepsilon t^{1-q}\right)= & F(t)+G(t)+u_{1}\left(t, \varepsilon t^{1-q}\right) \\
& +v_{1}\left(t, \varepsilon t^{1-q}\right),
\end{aligned}
$$

that is, the $H$-difference

$$
\left(F\left(t+\varepsilon t^{1-q}\right)+G\left(t+\varepsilon t^{1-q}\right)\right) \ominus(F(t)+G(t))=u_{1}\left(t, \varepsilon t^{1-q}\right)+v_{1}\left(t, \varepsilon t^{1-q}\right) .
$$

By similar reasoning, we get that there exist $u_{2}\left(t, \varepsilon t^{1-q}\right)$ and $v_{2}\left(t, \varepsilon t^{1-q}\right)$ such that

$$
\begin{aligned}
& F(t)=F\left(t-\varepsilon t^{1-q}\right)+u_{2}\left(t, \varepsilon t^{1-q}\right), \\
& G(t)=G\left(t-\varepsilon t^{1-q}\right)+v_{2}\left(t, \varepsilon t^{1-q}\right),
\end{aligned}
$$

and so

$$
\begin{aligned}
(F(t)+G(t))= & \left(F\left(t-\varepsilon t^{1-q}\right)+G\left(t-\varepsilon t^{1-q}\right)\right) \\
& +u_{2}\left(t, \varepsilon t^{1-q}\right)+v_{2}\left(t, \varepsilon t^{1-q}\right),
\end{aligned}
$$

that is, the $H$-difference

$$
\begin{gathered}
(F(t)+G(t)) \ominus\left(F\left(t-\varepsilon t^{1-q}\right)+G\left(t-\varepsilon t^{1-q}\right)\right) \\
=u_{2}\left(t, \varepsilon t^{1-q}\right)+v_{2}\left(t, \varepsilon t^{1-q}\right) .
\end{gathered}
$$

We observe that

$$
\begin{aligned}
& \lim _{\varepsilon \longrightarrow 0^{+}} \frac{u_{1}\left(t, \varepsilon t^{1-q}\right)}{\varepsilon}=\lim _{\varepsilon \longrightarrow 0^{+}} \frac{u_{2}\left(t, \varepsilon t^{1-q}\right)}{\varepsilon}=F^{(q)}(t), \\
& \lim _{\varepsilon \longrightarrow 0^{+}} \frac{v_{1}\left(t, \varepsilon t^{1-q}\right)}{\varepsilon}=\lim _{\varepsilon \longrightarrow 0^{+}} \frac{v_{2}\left(t, \varepsilon t^{1-q}\right)}{\varepsilon}=G^{(q)}(t) .
\end{aligned}
$$

Finally, by multiplying (21) and (24) with $1 / \varepsilon$ and passing to limit with $\lim _{\varepsilon \longrightarrow 0^{+}}$, we get that $F+G$ is $q$-differentiable and $T_{q}(F+G)(t)=T_{q} F(t)+T_{q} G(t)$. The case (ii) is similar to the previous one.

3.2. Fuzzy Fractional Integral. Let $q \in(0,1]$ and $F:(0, a) \longrightarrow \mathbb{R}_{\mathscr{F}}$ be such that $[F(t)]^{\alpha}=\left[f_{1}^{\alpha}(t), f_{2}^{\alpha}(t)\right]$ for all $t \in(0, a)$ and $\alpha \in[0,1]$. Suppose that $f_{1}^{\alpha}, f_{2}^{\alpha} \in C((0, a), \mathbb{R}) \cap L^{1}((0, a), \mathbb{R})$ for all $\alpha \in[0,1]$ and let 


$$
A_{\alpha}=:\left[\int_{0}^{t} \frac{f_{1}^{\alpha}}{x^{1-q}}(x) \mathrm{d} x, \int_{0}^{t} \frac{f_{2}^{\alpha}}{x^{1-q}}(x) \mathrm{d} x\right], \quad t \in(0, a) .
$$

Lemma 2. The family $\left\{A_{\alpha} ; \alpha \in[0,1]\right\}$, given by equation (26), defined a fuzzy number $F \in \mathbb{R}_{\mathscr{F}}$ such that $[F]^{\alpha}=A_{\alpha}$.

Proof. For $\alpha<\beta$, we have $f_{1}^{\alpha}(x) \leq f_{1}^{\beta}(x)$ and $f_{2}^{\alpha}(x) \geq f_{2}^{\beta}(x)$. It follows $A_{\alpha} \supseteq A_{\beta}$. Since $f_{1}^{0}(x) \leq f_{1}^{\alpha_{n}}(x) \leq f_{1}^{1}(x)$, we have

$$
\left|x^{q^{-1}} f_{i}^{\alpha_{n}}(x)\right| \leq \max \left\{a^{q^{-1}}\left|f_{i}^{0}(x)\right|, a^{q^{-1}}\left|f_{i}^{1}(x)\right|\right\}=: g_{i}(x),
$$

for $\alpha_{n} \in[0,1]$ and $i=1,2$. Obviously, $g_{i}$ is integrable on $(0, a)$. Therefore, if $\alpha_{n} \uparrow \alpha$, then by Lebesque's dominated convergence theorem, we have

$$
\lim _{n \longrightarrow \infty} \int_{0}^{t} \frac{f_{i}^{\alpha_{n}}}{x^{1-q}}(x) \mathrm{d} x=\int_{0}^{t} \frac{f_{i}^{\alpha}}{x^{1-q}}(x) \mathrm{d} x, \quad i=1,2 .
$$

From Theorem 1, the proof is complete.

Definition 4. Let $F \in C\left((0, a), \mathbb{R}_{\mathscr{F}}\right) \cap L^{1}\left((0, a), \mathbb{R}_{\mathscr{F}}\right)$ define the fuzzy fractional integral for $q \in(0,1]$,

$$
I_{q}(F)(t)=I\left(t^{q-1} F\right)(t)=\int_{0}^{t} \frac{F}{x^{1-q}}(x) \mathrm{d} x,
$$

by’

$$
\begin{aligned}
{\left[I_{q}(F)(t)\right]^{\alpha} } & =\left[I\left(t^{q-1} F\right)(t)\right]^{\alpha}=\left[\int_{0}^{t} \frac{F}{x^{1-q}}(x) \mathrm{d} x\right]^{\alpha} \\
& =\left[\int_{0}^{t} \frac{f_{1}^{\alpha}}{x^{1-q}}(x) \mathrm{d} x, \int_{0}^{t} \frac{f_{2}^{\alpha}}{x^{1-q}}(x) \mathrm{d} x\right],
\end{aligned}
$$

where the integral $\int_{0}^{t}\left(f_{i}^{\alpha} / x^{1-q}\right)(x) \mathrm{d} x$ for $i=1,2$ is the usual Riemann improper integral. Also, the following properties are obvious.

Lemma 3. Let $q \in(0,1]$ and $F, G:(0, a) \longrightarrow \mathbb{R}_{\mathscr{F}}$ be fractional integrable and $\lambda \in \mathbb{R}$. Then,

(i) $I_{q} \lambda F(t)=\lambda I_{q} F(t)$

(ii) $I_{q}(F+G)(t)=I_{q} F(t)+I_{q} G(t)$

Proof. The proof is similar to the proof of Theorem 4.3 cases (i) and (ii) in [2] and is omitted.

Theorem 8. $T_{q} I_{q}(F)(t)=F(t)$, for $t \geq 0$, where $F$ is any continuous function in the domain of $I_{q}$

Proof. Since $F$ is continuous, then $I_{q}(F)(t)$ is clearly $q$-differentiable because

$$
I_{q}(F)(t)=I\left(t^{q-1} F\right)(t),
$$

and $t^{q-1} F(t)$ is continuous for all $t \in(0, a)$; then, by Theorem 5.6 in [2] and Theorem 6, the fractional integral is $q$-differentiable. Hence,

$$
\begin{aligned}
{\left[T_{q} I_{q}(F)(t)\right]^{\alpha} } & =\left[t^{1-q} \frac{\mathrm{d}}{\mathrm{d} t} I_{q}(F)(t)\right]^{\alpha} \\
& =\left[t^{1-q} \frac{\mathrm{d}}{\mathrm{d} t} \int_{0}^{t} \frac{f_{1}^{\alpha}(x)}{x^{1-q}} \mathrm{~d} x, t^{1-q} \frac{\mathrm{d}}{\mathrm{d} t} \int_{0}^{t} \frac{f_{2}^{\alpha}(x)}{x^{1-q}} \mathrm{~d} x\right] \\
& =\left[t^{1-q} \frac{f_{1}^{\alpha}(t)}{t^{1-q}}, t^{1-q} \frac{f_{2}^{\alpha}(t)}{t^{1-q}}\right] \\
& =[F(t)]^{\alpha} .
\end{aligned}
$$

Theorem 9. Let $q \in(0,1]$ and $F$ be $q$-differentiable in $(0, a)$, and assume that the conformable derivative $F^{(q)}$ is integrable over $(0, a)$. Then, for each $s \in(0, a)$, we have

$$
F(s)=F(0)+I_{q} F^{(q)} \text {. }
$$

Proof. Let $q \in(0,1]$ and $\alpha \in[0,1]$ be fixed. We shall prove that

$$
F_{\alpha}(s)=F_{\alpha}(0)+I_{q} F_{\alpha}^{(q)},
$$

where $F_{\alpha}^{(q)}$ is the Hukuhara conformable fractional derivative of $F_{\alpha}$; then, using Theorems 3 and 6 gives us the following equation.

$$
\begin{aligned}
F_{\alpha}(s) & =F_{\alpha}(0)+I_{q} F_{\alpha}^{(q)} \\
& =F_{\alpha}(0)+I_{q}\left(t^{1-q} F_{\alpha}^{\prime}\right) .
\end{aligned}
$$

By equation (29), we have

$$
\begin{aligned}
F_{\alpha}(s) & =F_{\alpha}(0)+I_{q}\left(t^{1-q} F_{\alpha}^{\prime}\right) \\
& =F_{\alpha}(0)+\int_{0}^{s} t^{q-1}\left(t^{1-q} F_{\alpha}^{\prime}\right) .
\end{aligned}
$$

So,

$$
F_{\alpha}(s)=F_{\alpha}(0)+\int_{0}^{s} F_{\alpha}^{\prime},
$$

where $F_{\alpha}^{\prime}$ is the Hukuhara derivative of $F_{\alpha}$; equation (37) is also true for a fuzzy mapping $F:(0, a) \longrightarrow \mathbb{R}_{\mathscr{F}}$. The equality (34) now follows Theorem 5.7 in [2].

\section{Fuzzy Comformable Fractional Differential Equations}

We study the fuzzy initial value problem

$$
\begin{aligned}
T_{q} x(t) & =F(t, x(t)), \quad q \in(0,1], \\
x(0) & =x_{0},
\end{aligned}
$$

where $F:(0, a) \times \mathbb{R}_{\mathscr{F}} \longrightarrow \mathbb{R}_{\mathscr{F}}$ is the continuous fuzzy mapping, and $x_{0}$ is the fuzzy number. From Theorems 5,8 , and 9, it immediately follows. 
Theorem 10. A mapping $x:(0, a) \longrightarrow \mathbb{R}_{\mathscr{F}}$ is a solution to problem (38) if and only if it is continuous and satisfies the integral equation:

$$
x(t)=x_{0}+I_{q} F(t, x(t)),
$$

for all $t \in(0, a)$ and $q \in(0,1]$.

Theorem 11. Let $F:(0, a) \times \mathbb{R}_{\mathscr{F}} \longrightarrow \mathbb{R}_{\mathscr{F}}$ be continuous, and assume that there exists $k>0$ such that

$$
d(F(t, x), F(t, y)) \leq k d(x, y),
$$

for all $t \in(0, a), x, y \in \mathbb{R}_{\mathscr{F}}$. Then, problem (38) has a unique solution on $(0, a)$.

Proof. If in problem (38) we consider the conformable derivative $x^{(q)}$ for all $q \in(0,1]$ Theorem 3 , then from Theorem 6.1 in [2] and using Definition 4 and Lemma $1,(0, a)$ we can prove that there exists an unique solution on $(0, a)$, and the proof is now complete.

Remark 2. In [15], it is observed that if we fuzzify the equivalent ordinary differential equation $x^{(q)}+x=0$, then we will get fuzzy differential equations (the equation was fuzzified by adding a forcing term $\sigma(t)$ in the right-hand side). That is, if we consider fuzzy differential equation $x^{(q)}+$ $x=\sigma(t)$ with the same initial condition $x\left(t_{0}\right)=x_{0}$, we get the result.

Consider the following linear fractional equation:

$$
x^{(q)}(t)+x(t)=\sigma(t), \quad q \in(0,1] \text { and } t \in(0, a),
$$

where $\sigma \in C\left((0, a) \times \mathbb{R}_{\mathscr{F}}\right)$. Denote $[x(t)]^{\alpha}=\left[x_{1}^{\alpha}(t), x_{2}^{\alpha}(t)\right]$, $\left[x_{0}\right]^{\alpha}=\left[x_{01}^{\alpha}, y_{02}^{\alpha}\right]$, and $[\sigma(t)]^{\alpha}=\left[\sigma_{1}^{\alpha}(t), \sigma_{2}^{\alpha}(t)\right]$.

Theorem 12. Equation (41) has a unique solution in $(0, a)$, and for given initial $x_{0} \in \mathbb{R}_{\mathscr{F}}$, it is given by

$$
\begin{aligned}
x(t) & =x_{0} e^{-\left(t^{q} / q\right)}+\int_{0}^{t} s^{q-1} \sigma(s) e^{\left(s^{q}-t^{q}\right) / q} \mathrm{~d} s, \quad t \in(0, a), \\
& =x_{0} e^{-\left(t^{q} / q\right)}+e^{-\left(t^{q} / q\right)} I_{q}\left(\sigma(t) e^{\left(t^{q} / q\right)}\right), \quad t \in(0, a) .
\end{aligned}
$$

Proof. Equation (41) can be written, levelwise, as

$$
\begin{aligned}
& {\left[\left(x_{1}^{\alpha}\right)^{(q)}(t),\left(x_{2}^{\alpha}\right)^{(q)}(t)\right]+\left[x_{1}^{\alpha}(t), x_{2}^{\alpha}(t)\right]} \\
& =\left[\sigma_{1}^{\alpha}(t), \sigma_{2}^{\alpha}(t)\right], \quad t \in(0, a),
\end{aligned}
$$

for every $\alpha \in[0,1]$, so that

$$
\begin{aligned}
& \left(x_{1}^{\alpha}\right)^{(q)}(t)+x_{1}^{\alpha}(t)=\sigma_{1}^{\alpha}(t), \\
& \left(x_{2}^{\alpha}\right)^{(q)}(t)+x_{2}^{\alpha}(t)=\sigma_{2}^{\alpha}(t) .
\end{aligned}
$$

Thus, for $t \in(0, a)$,

$$
\begin{aligned}
& \left(x_{1}^{\alpha} e^{\left(t^{q} / q\right)}\right)^{(q)}(t)=\sigma_{1}^{\alpha}(t) e^{\left(t^{q} / q\right)}, \\
& \left(x_{2}^{\alpha} e^{\left(t^{q} / q\right)}\right)^{(q)}(t)=\sigma_{2}^{\alpha}(t) e^{\left(t^{q} / q\right)},
\end{aligned}
$$

and, therefore, it can be deduced that

$$
\begin{aligned}
& x_{1}^{\alpha}(t)=x_{01}^{\alpha} e^{-\left(t^{q} / q\right)}+\int_{0}^{t} s^{q-1} \sigma_{1}^{\alpha}(s) e^{\left(s^{q}-t^{q}\right) / q} \mathrm{~d} s, \\
& x_{2}^{\alpha}(t)=x_{02}^{\alpha} e^{-\left(t^{q} / q\right)}+\int_{0}^{t} s^{q-1} \sigma_{2}^{\alpha}(s) e^{\left(s^{q}-t^{q}\right) / q} \mathrm{~d} s .
\end{aligned}
$$

This proves that, for $\alpha \in[0,1]$,

$$
\begin{aligned}
{[x(t)]^{\alpha}=} & {\left[x_{0}\right]^{\alpha} e^{-\left(t^{q} / q\right)} } \\
& +\int_{0}^{t} s^{q-1}[\sigma(s)]^{\alpha} e^{\left(s^{q}-t^{q}\right) / q} \mathrm{~d} s, \quad t \in(0, a) .
\end{aligned}
$$

So,

$$
\begin{aligned}
{[x(t)]^{\alpha}=} & {\left[x_{0}\right]^{\alpha} e^{-\left(t^{q} / q\right)} } \\
& +e^{-\left(t^{q} / q\right)} I_{q}\left([\sigma(t)]^{\alpha} e^{\left(t^{t / q}\right)}\right), \quad t \in(0, a) .
\end{aligned}
$$

\section{Conclusion}

In this study, for developing and proving some results for fuzzy conformable differentiability and fuzzy fractional integrability of such functions, we provided existence and uniqueness solutions to fuzzy fractional problems for order $q \in(0,1]$ FFDEs, which is interpreted by using the generalized conformable fractional derivatives concept.

For future research, we will solve the fractional fuzzy conformable partial differential equations $[22,23]$ and a class of linear differential dynamical systems [24] by using the proposed method.

\section{Data Availability}

The data used to support the findings of this study are available from the corresponding author upon request.

\section{Conflicts of Interest}

The authors declare that they have no conflicts of interest.

\section{References}

[1] M. L. Puri and D. A. Ralescu, "Differentials of fuzzy functions," Journal of Mathematical Analysis and Applications, vol. 91, no. 2, pp. 552-558, 1983.

[2] O. Kaleva, "Fuzzy differential equations," Fuzzy Sets and Systems, vol. 24, no. 3, pp. 301-317, 1987.

[3] O. Kaleva, "A note on fuzzy differential equations," Nonlinear Analysis: Theory, Methods \& Applications, vol. 64, no. 5, pp. 895-900, 2006.

[4] R. P. Agarwal, V. Lakshmikantham, and J. J. Nieto, "On the concept of solution for fractional differential equations with uncertainty," Nonlinear Analysis: Theory, Methods \& Applications, vol. 72, no. 6, pp. 2859-2862, 2010. 
[5] M. Alabedalhadi, M. Al-Smadi, S. Al-Omari, D. Baleanu, and S. Momani, "Structure of optical soliton solution for nonliear resonant space-time Schrödinger equation in conformable sense with full nonlinearity term," Physica Scripta, vol. 95, no. 10, Article ID 105215, 2020.

[6] M. Al-Smadi, O. A. Arqub, and S. Momani, "Numerical computations of coupled fractional resonant Schrodinger equations arising in quantum mechanics under conformable fractional derivative sense," Physica Scripta, vol. 95, no. 7, Article ID 075218, 2020.

[7] S. Arshad and V. Lupulescu, "On the fractional differential equations with uncertainty," Nonlinear Analysis: Theory, Methods \& Applications, vol. 74, no. 11, pp. 3685-3693, 2011.

[8] A. Harir, S. Melliani, L. S. Chadli, and E. Minchev, "Solutions of fuzzy fractional heat-like and wave-like equations by variational iteration method," International Journal of Contemporary Mathematical Sciences, vol. 15, no. 1, pp. 11-35, 2020.

[9] A. Harir, S. Melliani, and L. S. Chadli, "Fuzzy fractional evolution equations and fuzzy solution operators," Advances in Fuzzy Systems, vol. 2019, Article ID 5734190, 10 pages, 2019.

[10] A. Harir, S. Melliani, and L. S. Chadli, "Fuzzy conformable fractional semigroups of operators," International Journal of Differential Equations, vol. 2020, Article ID 8836011, 6 pages, 2020.

[11] Z. Shuqin, "Monotone iterative method for initial value problem involving Reimann Liouville fractional derivatives," Nonlinear Analysis, vol. 71, pp. 2087-2093, 2009.

[12] G. Gumah, S. Al-Omari, and D. Baleanu, "Soft computing technique for a system of fuzzy Volterra integro-differential equations in a Hilbert space," Applied Numerical Mathematics, vol. 152, pp. 310-322, 2020.

[13] G. Gumah, M. F. M. Naser, M. Al-Smadi, S. K. Q. Al-Omari, and D. Baleanu, "Numerical solutions of hybrid fuzzy differential equations in a Hilbert space," Applied Numerical Mathematics, vol. 151, pp. 402-412, 2020.

[14] C. Y. Ma, B. Shiri, G. C. Wu, and D. Baleanu, "New fractional signal smoothing equations with short memory and variable order," Optik, vol. 218, Article ID 164507, 2020.

[15] R. Khalil, M. Al Horani, A. Yousef, and M. Sababheh, "A new definition of fractional derivative," Journal of Computational and Applied Mathematics, vol. 264, pp. 65-70, 2014.

[16] O. A. Arqub and M. Al-Smadi, "Fuzzy conformable fractional differential equations: novel extended approach and new numerical solutions," Soft Computing, vol. 24, no. 16, pp. 12501-12522, 2020.

[17] A. Harir, S. Melliani, and L. S. Chadli, "Fuzzy generalized conformable fractional derivative," Advances in Fuzzy Systems, Advances in Fuzzy Systems, vol. 2019, Article ID 1954975, 7 pages, 2020.

[18] H. Y. Goo and J. S. Park, "On the continuity of the Zadeh extensions," Journal of the Chungcheong Mathematical Society, vol. 20, no. 4, pp. 525-533, 2007.

[19] S. Markov, "Calculus for interval functions of a real variable," Computing, vol. 22, no. 4, pp. 325-337, 1979.

[20] L. Stefanini, "A generalization of Hukuhara difference and division for interval and fuzzy arithmetic," Fuzzy Sets and Systems, vol. 161, no. 11, pp. 1564-1584, 2010.

[21] G. A. Anastassiou and S. G. Gal, "On a fuzzy trigonometric approximation theorem of weierstrasstype," Journal of Fuzzy Mathematics, vol. 9, pp. 701-708, 2001.

[22] M. Al-Smadi, O. Abu Arqub, and S. Hadid, "An attractive analytical technique for coupled system of fractional partial differential equations in shallow water waves with conformable derivative," Communications in Theoretical Physics, vol. 72, no. 8, Article ID 085001, 2020.

[23] M. Al-Smadi, "Numerical simulation of conformable fuzzy differential equations," in Mathematical Methods and Modelling in Applied Sciences. ICMRS 2019. Lecture Notes in Networks and Systems, M. Zeki Sarikaya, H. Dutta, A. Ocak Akdemir, and H. Srivastava, Eds., Springer, Cham, Switzerland, vol. 123, 2020.

[24] A. Harir, S. Melliani, H. El Harfi, and L. S. Chadli, "Variational iteration method and differential transformation method for solving the SEIR epidemic model," International Journal of Differential Equations, vol. 2020, Article ID 3521936, 7 pages, 2020.

[25] S. Seikkala, "On the fuzzy initial value problem," Fuzzy Sets and Systems, vol. 24, no. 3, pp. 319-330, 1987. 